\title{
An interferometric radar sensor for monitoring the vibrations of structures at short ranges
}

\author{
Guido Luzi ${ }^{1 *}$, Michele Crosetto ${ }^{1}$, Eduard Angelats ${ }^{1}$, Enric Fernández ${ }^{1}$ \\ ${ }^{1}$ Centre Tecnòlogic de Telecomunicacions de Catalunya, CTTC/CERCA, 08860 Castelldefels (BCN), Spain
}

\begin{abstract}
The Real-Aperture-Radar (RAR) interferometry technique consolidated in the last decade as an operational tool for the monitoring of large civil engineering structures as bridges, towers, and buildings. In literature, experimental campaigns collected through a well-known commercial equipment have been widely documented, while the cases where different types of sensors have been tested are a few. On the bases of some experimental tests, a new sensor working at high frequency, providing some improved performances, is here discussed. The core of the proposed system is an off-the-shelf, linear frequency modulated continuous wave device. The development of this apparatus is aimed at achieving a proof-of-concept, tackling operative aspects related to the development of a low cost and reliable system. The capability to detect the natural frequencies of a lightpole has been verified; comparing the results of the proposed sensor with those ones obtained through a commercial system based on the same technique, a more detailed description of the vibrating structure has been achieved. The results of this investigation confirmed that the development of sensors working at higher frequencies, although deserving deeper studies, is very promising and could open new applications demanding higher spatial resolutions at close ranges.
\end{abstract}

\section{Introduction}

The use of interferometric radar sensors, namely RealAperture-Radar (RAR) interferometry, consolidated in the last decade as an operational tool for the monitoring of large civil engineering structures as bridges, towers, and buildings: for an exhaustive bibliography on the topic see [1]. Although the accuracy of a radar estimate of displacement, usually of the order of tens of microns, cannot be comparable with that of optical systems, as Laser Doppler vibrometers, a microwave sensor is able to provide data with a negligible effect of the atmospheric propagation, weather conditions, and from distance up to one kilometre. Furthermore an interferometric radar, with respect to velocimeters, provides directly estimates of the displacement of the vibrating object, allowing to obtain direct inputs for modal analysis. The main limitation of this technique is that only the component along the radartarget line of sight (LOS) can be measured. The spatial resolution available for the target monitoring are mainly dictated by radar parameters as the swept bandwidth, and the achievable signal to noise ratio (SNR), which affect the range resolution, i.e. the capability to distinguish two different targets or parts of a surface, and the available accuracy. In literature, data collected through a wellknown commercial apparatus working at $\mathrm{Ku}$ band are well documented, while the cases where different sensors have been tested are a few. The most popular system, namely Ibis-STM, is manufactured by the IDS (Ingegneria dei Sistemi $\mathrm{SpA}$ ) company, and is available in the market. The system was born more than ten years ago, and it represents a trade-off after some experimental pioneer tests carried out during this period.

In the last years the costs of microwave technology at higher frequencies drastically lowered, and the performances of the on-the shelf devices have strongly improved. More recently, in the last few years, new prototypes have been presented in literature (see for example [2-6]); systems with different characteristics, operating at different bands have been developed to improve the performances of Ibis-S and aiming at reducing the costs of the apparatus. On the bases of some basic experiments, a novel sensor working at a higher frequency, providing some improved performances, has been developed at CTTC; in this paper some preliminary results are discussed. The core of the proposed system is an off-the-shelf, linear frequency modulated continuous wave (FMCW) K-band (centre frequency: $24 \mathrm{GHz}$ ) sensor. This device can provide a lower maximum operating range in comparison with respect to the commercial available systems, but a better range resolution. The development of this system is devoted to a proof-of-concept aimed at tackling operative aspects, including a reduction of the costs. In the present version, the data acquired through the radar are processed in two steps and not in real time. The main test carried out at CTTC is focused to evaluate its capability to detect the first natural frequency of a lightpole, comparing the results of the novel sensor, from here on referred as "NeoRAR", with those ones obtained through Ibis-S. An outstanding outcome of this test is the achievement of a

\footnotetext{
* Corresponding author: gluzi@,cttc.es
} 
better range resolution, and hence a more detailed description of the vibrating structure.

The paper contains a brief introduction of the working principle followed by a recall of the main features of a commercial microwave interferometer. The novel apparatus is then described and some experimental results are discussed.

\subsection{The working principle}

Radar is a time of flight device, which uses the time, elapsed between the transmitting and the receiving of an electromagnetic waveform, to locate targets included in the illuminated area. The basic output from a radar survey is a 1D signal, representing the amplitude of the echoes coming from the target included in the field of view (FOV) of the radar antenna; this signal is usually called "range profile" because its different peaks correspond to contributions coming from targets located at different distances. Standard radar is based on the transmission of signals composed of temporal periodic pulses of the amplitude. In a different way radar interferometers, which need coherent signal detection, are usually based on the frequency modulation of the transmitted signal, and specific waveforms sweeping a finite band composed of different frequencies, B, (Frequency Modulated Continuous Wave, FMCW, or Step Frequency Continuous Wave, SFCW) are used to assure coherent signals. The coherence of the radar sensor allows interferometric processing providing the capability of a sub-meter range resolution [7]. When different targets are present, the radar is able to provide their displacement history. The characteristics of the used antennas determine the volume of a spatial cell of the radar measurement, usually called radar bin.

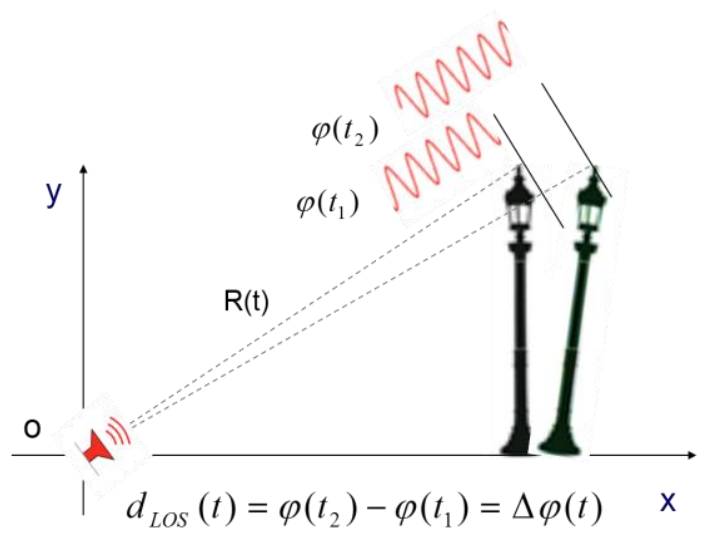

Fig. 1. A scheme of the working principle. dLos is the projection of the displacement along the radar line of site.

The use of interferometric radar to detect the vibration of an object is based on the capability of a coherent radar working at microwave frequency, to measure variations in time of the differential phase of the received echo with respect to the transmitted signal. As simply shown in fig. 1 the variation of the LOS distance can be measured calculating the temporal variation of the interferometric phase. A higher operating frequency means in general a higher sensitivity to displacement variation. Considering that presently available apparatus uses a wavelength of the order of two centimetres, we can appreciate variations down to tens of microns. The vibration is seen by the radar as a range variation between the radar and the vibrating objects. The achievable accuracy is mainly determined by the signal to noise ratio of the acquisition which, in turn, depends on the intensity of the reflected signal. The radar reflecting properties of a target, the transmitted power, the distance, geometric factors (shape and orientation) and finally the dielectric characteristics of the target are the main factors affecting the strength of the received signal and hence the accuracy of the phase measurement.

\subsection{A commercial system}

The CTTC owns a commercial radar with interferometric capability: the IBIS-STM, manufactured and marketed by IDS [8]. The system consists of a sensor module, a control PC and a power supply unit and data processing software. A summary of its main characteristics is reported in Table 1 . It operates at $\mathrm{Ku}$ band $(17 \mathrm{GHz})$, i.e. with a corresponding wavelength lower than 2 centimetres, and with a maximum band of $300 \mathrm{MHz}$ (model available in Europe). The system can be used with various antennas to better adapt the measuring conditions to different geometries and monitored surfaces. The radar has been used since many years by many researches to carry out monitoring different structures This apparatus has been used to measure the dynamic response of a variety of structures, such as bridges [9], wind farms [10], buildings [11] and towers [12], [13].

Table 1. Ibis-S Characteristics.

\begin{tabular}{|l|l|}
\hline \multicolumn{1}{|c|}{ Parameters } & \multicolumn{1}{c|}{ Value } \\
\hline Operating frequency & $17.2 \mathrm{GHz}(\mathrm{Ku}$ band $)$ \\
\hline Bandwidth (CWSF) & $300 \mathrm{MHz}$ \\
\hline $\begin{array}{l}\text { Max. operational } \\
\text { distance }\end{array}$ & $1000 \mathrm{~m}$ \\
\hline Range resolution & $0.5 \mathrm{~m}$ \\
\hline Nominal sensitivity & $0.00001 \mathrm{~m}$ \\
\hline Max. acquisition rate & $200 \mathrm{~Hz}$ \\
\hline Polarization & Linear $(\mathrm{HH}$ or $\mathrm{VV})$ \\
\hline
\end{tabular}

\section{The novel apparatus}

\subsection{The radar sensor}

The transceiver used to implement the radar sensor here discussed, is based on the use of a on the shelf device marketed by Sivers IMA AB (Sweden). Its main characteristics are resumed in Table 2 . With respect to Ibis-STM, the operating frequency is higher, and its 
maximum bandwidth is larger, $1500 \mathrm{MHz}$ in comparison to $300 \mathrm{MHz}$; this allows achieving a nominal range resolution of $10 \mathrm{~cm}$. It is a monostatic radar with a $20 \mathrm{~dB}$ standard gain horn antenna. The system is powered by a single $12 \mathrm{~V}$ battery.

In fig. 2, a picture of the system, including the RF sensor mounted on a small tripod, a laptop and a battery, is shown. In the present configuration, the sampling frequency is $3.5 \mathrm{~Hz}$ but we expect to increase it up to 12 $\mathrm{Hz}$

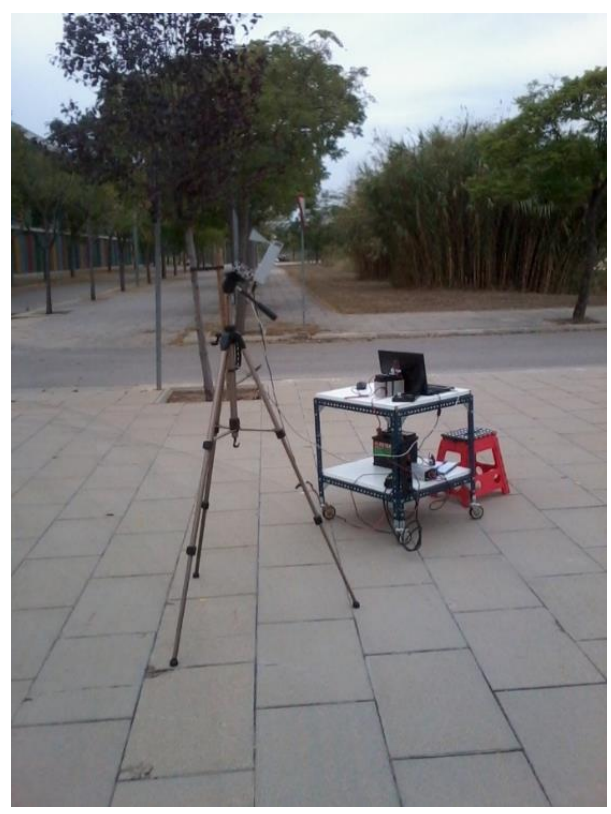

Fig. 2. Picture of the NeoRAR system.

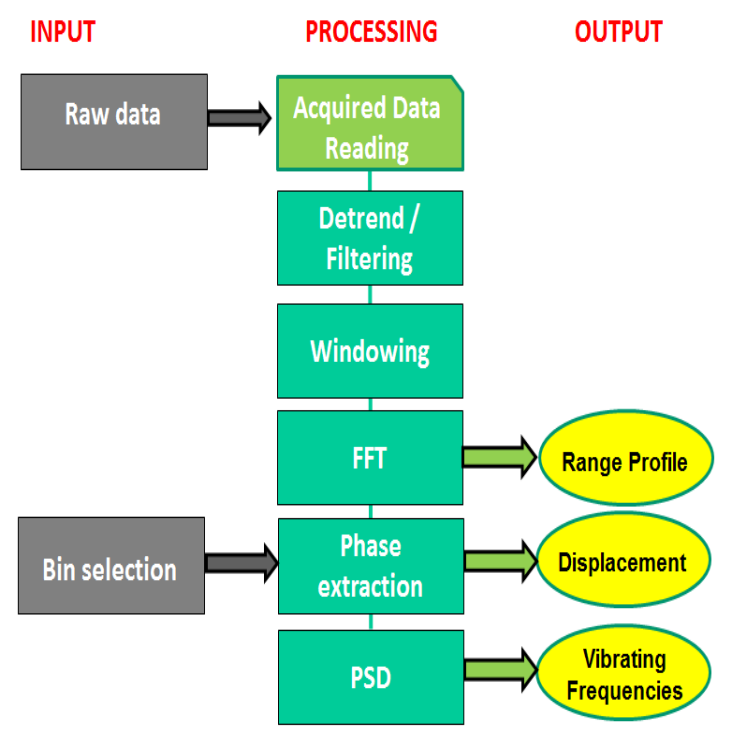

Fig. 3. A scheme of the data processing chain used to elaborate the NeoRAR data

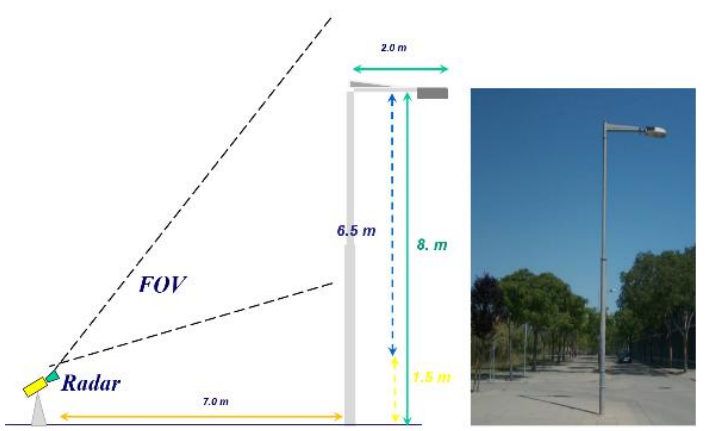

Fig. 4. A scheme and a picture of the target used for testing the sensor.

Table 2. Sivers IMA transceiver sensor characteristics.

\begin{tabular}{|l|l|}
\hline \multicolumn{1}{|c|}{ Parameters } & \multicolumn{1}{c|}{ Value } \\
\hline Operating frequency & $24.75 \mathrm{GHz}(\mathrm{K}$ band $)$ \\
\hline Bandwidth $(\mathrm{CWFM})$ & $1500 \mathrm{MHz}$ \\
\hline Max. operational distance & $75 \mathrm{~m}$ \\
\hline Range resolution & $0.1 \mathrm{~m}$ \\
\hline Max. acquisition rate & $3.5 \mathrm{~Hz}$ \\
\hline Polarization & $\mathrm{VV}$ \\
\hline Battery Autonomy & $12 \mathrm{~h}$ \\
\hline Antenna Field of View $\left(^{\circ}\right) \mathrm{H}$ & 18.6 \\
\hline Antenna Field of View $\left(^{\circ}\right) \mathrm{E}$ & 16.1 \\
\hline
\end{tabular}

\subsection{The data processing}

The data acquired through radar are usually processed in two steps: the one necessary to transform the radar data to a range profile where we can identity the different parts of the monitored surface or target. This step is based on an inverse Fourier transform after a previous filtering and windowing of the raw signal. In particular a Kaiser function is used for windowing the signal before the DFT, and a high pass Butterworth filter of third order, with 0.1 $\mathrm{Hz}$ cut-off frequency, is applied. The second step consists in the extraction of the phase temporal series and their spectral analysis. DFT or power spectral density of the displacement stories are usually calculated to detect the main frequencies. In fig. 3 a scheme of the processing steps is shown.

\section{Experimental Results}

\subsection{The target}

The target selected for the test of the novel sensor performances is a metal lightpole located in the Parc Mediterrani de la Tecnologia of Castelldefels (Spain), where CTTC is located. In fig. 5a, a picture and a scheme of the size of the target are shown. The measurements were carried out on $29^{\text {th }}$ September 2016; the day was affected by irregular wind shears. Micro tremor and wind are the main causes of target vibration; vehicular traffic in this area is very sporadic and it was absent during the data 
acquisition. A first test consisted in verifying the actual range resolution: moving the NeoRAR of known displacement steps, a $10 \mathrm{~cm}$ value was obtained as expected using the whole $1500 \mathrm{MHz}$ bandwidth. The results of this test are shown in fig. 5 where the displacement obtained from the bin identification and the target displacement are compared.
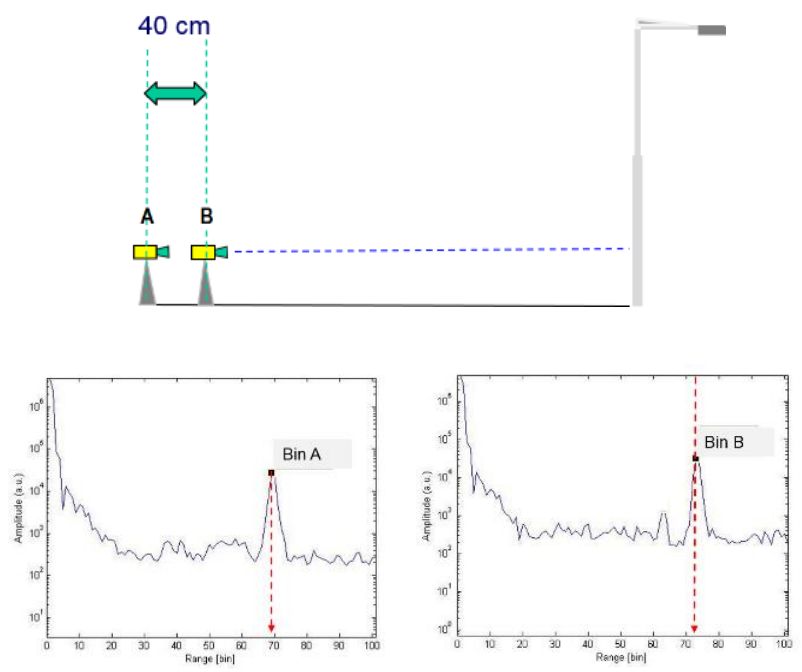

Fig. 5. Test to verify the range resolution of the NeoRAR: a displacement of $40 \mathrm{~cm}$ corresponds to a 4 bins $(\mathrm{Bin} A=69$, $\mathrm{Bin} B=73$ ) variation.

\subsection{The data acquisition}

To test the capability in vibrations monitoring of the proposed system, data acquisitions have been acquired in front of the lightpole with the geometry shown in fig. 4 to sample different parts of the pole. In fig. 6 we show the section of the range profile where four bins corresponding to different heights of the lightpole are marked. To retrieve the distance from the radar bin number note that each bin to a $0.1 \mathrm{~m}$ step, and due to an instrumental constant, we have to take off an offset (3 bins).

Table 3. Bin identification and detected frequencies obtained from the NeoRAR monitoring.

\begin{tabular}{|c|c|c|c|}
\hline Bin & Range(m) & $\begin{array}{l}\text { Corresponding } \\
\text { Height (m) }\end{array}$ & $\begin{array}{l}\text { Detected } \\
\text { Frequency (Hz) }\end{array}$ \\
\hline 76 & 7.3 & 4.2 & 1.512 \\
\hline 88 & 8.5 & 6.8 & 1.519 \\
\hline 93 & 9.0 & 7.6 & 1.519 \\
\hline 95 & 9.2 & 7.8 & $0.467 / 1.512$ \\
\hline
\end{tabular}

\subsection{Data analysis}

In fig. 7 we show the spectral response obtained processing the LOS displacements corresponding to the four bins identified in fig. 6. The amplitude of the displacement increases with the height, as expected for the first vibrating mode of the pole. In table 3 the height of the lightpole part sampled by the radar measurement is also reported. These results can be compared to those obtained from the monitoring of the same lightpole carried out using Ibis-S only two bins were analysed, as can be seen in fig. 8 .

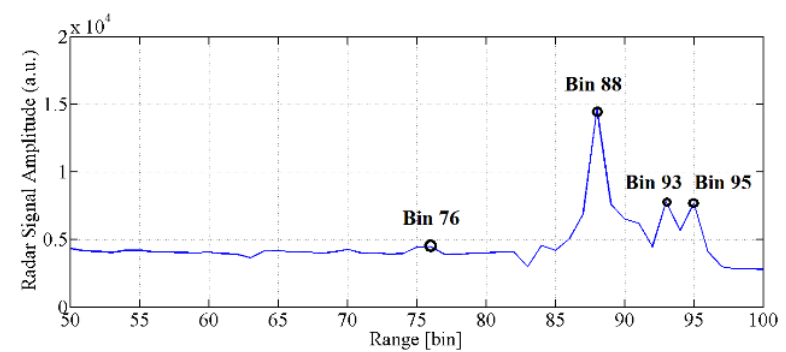

Fig. 6 Range profile and bin identification.
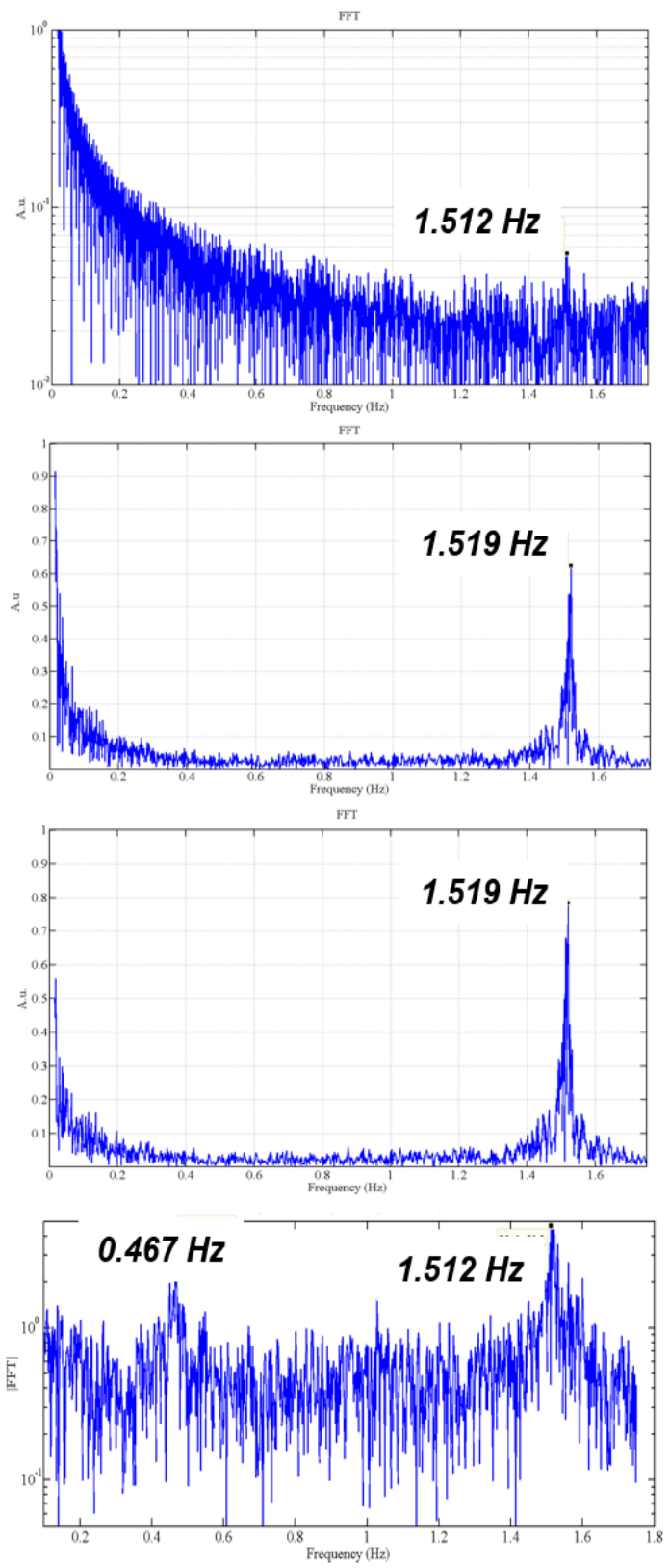

Fig. 7 FFT calculated from the displacement samples obtained from the four selected bins; from top to bottom: Bin76, Bin88, Bin94 and Bin95. In the first and the last plots logarithmic y axis scale is used to improve the peaks' detection. 


\section{Conclusions}

In this paper after introducing the basic of radar interferometry applied to detect civil structures' vibrating frequencies, preliminary results of a test aimed at testing a novel microwave interferometer have been discussed. On the bases of a simple experimental test, the main performances of a new FMCW sensor, working at $\mathrm{K}$ band (24 GHz), as a monitoring tool of mechanical vibration, have been analysed. Results obtained through the new sensor are in good agreement with that detected by the Ibis-S measurement: the power spectral density obtained from Ibis-S monitoring is shown in fig. 8. In addition, the higher spatial resolution available from NeoRAR allowed obtaining of a second frequency not detected by Ibis-S. The analysed sensor is very promising and deserves deeper studies.

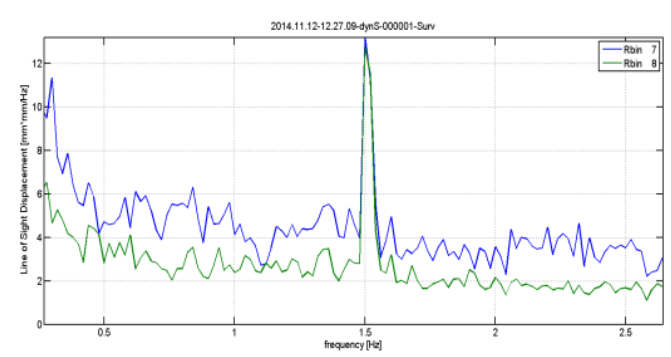

Fig. 8 LOS displacement PSD calculated from data acquired through Ibis-S.

\section{References}

1. M. Pieraccini, Monitoring of Civil Infrastructures by Interferometric Radar: A Review. The Scientific World Journal (2013), Article ID 786961, http://dx.doi.org/10.1155/2013/786961. (2013)

2. G. Grazzini., M. Pieraccini, D. Dei, C. Atzeni, Simple Microwave sensor for remote detection of structural vibration. Electronics Letters 2009, 45 11, 567-569 (2009).

3. J.Abril, E. Nova., A. Broquetas, A. Aguasca, J. Romeu, L. Jofre, Micrometric deformation imaging at W-Band". In Proceedings of the 2011 IEEE International Microwave Workshop. IMWS 2011. pp. 65-68 (2011).

4. F. Papi, N. Donati, M. Pieraccini, Handy Microwave Sensor for Remote Detection of Structural Vibration. EWSHM - 7th European Workshop on Structural Health Monitoring, Jul 2014, Nantes, France (2014)

5. Li Cunlong, Chen Weimin, Liu Gang, Yan Rong, Xu Hengyi and Qi Yi, A Noncontact FMCW Radar Sensor for Displacement Measurement in
Structural Health Monitoring. Sensors 2015, 15 7412-7433; doi:10.3390/s150407412. (2015)

6. G. Giunta, A.Monti-Guarnieri, D. D.'Aria, F.Speziali, P.Falcone, L.Maggi, G. Amoroso, A novel technique for very accurate threedimensional monitoring of structural vibrations and displacement by remote radar sensors, Radar Conference IET. (2015)

7. C. Gentile, G. Bernardini. An interferometric radar for non-contact measurement of deflections on civil engineering structures: laboratory and full-scale tests. Structure and Infrastructure Engineering $\mathbf{6}, 521-534$. DOI: 10.1080/15732470903068557 (2010)

8. F. Coppi, C. Gentile, P. Ricci, A software tool for processing the displacement time series extracted from raw radar data. Proceedings of the 9th Int. Conference on Vibration Measurements by Laser and non-contact Techniques, Ancona, Italy; 22-25 June 2010. AIP Conference Proceedings 1253, ed. E.P. Tomasini (2010)

9. M. Pieraccini, M. Fratini, F. Parrini, C. Atzeni, Dynamic monitoring of bridges using a highspeed coherent radar. 2006, IEEE TGRS, 40 11, 3284-3288 (2006)

10. M. Pieraccini, F. Parrini, M. Fratini, C. Atzeni, P. Spinelli. In-service testing of wind turbine towers using a microwave sensor. Renewable Energy 2008, 33, 1 pp. 13-21, ISSN:0960-1481 URL:http://dx.medra.org/10.1016/j.renene.200 7.02.001 (2008)

11. C. Negulescu, G. Luzi, M. Crosetto, D. Raucoules, A. Roullé, D. Monfort, L. Pujades, B. Colas, T. Dewez, Comparison of seismometer and radar measurements for the modal identification of civil engineering structures. Engineering Structures, 51, 10-22 (2013)

12. G. Luzi, M. Crosetto, M. Cuevas-González, A radar-based monitoring of the Collserola Tower (Barcelona). Mechanical Systems and Signal Processing 2014, 49, 234-248 (2014)

13. C. Atzeni, A. Bicci, D. Dei, M. Fratini, M. Pieraccini. Remote survey of the leaning tower of Pisa by interferometric sensing. IEEE GRSL 2010, 7 1, 185-189. 